\title{
Desenvolvimento Docente nos Cursos de Medicina: um Campo Fecundo
}

\author{
Faculty Development Courses in Medicine: \\ A Fruitful Field
}

Cinthia Rêgo Sylvia Helena Batista

\author{
PALAVRAS-CHAVE \\ - Aprendizagem Baseada \\ em Problemas. \\ - Capacitação \\ - Escolas Médicas. \\ - Docentes de Medicina. \\ - Educação Médica.
}

\section{KEYWORDS \\ - Problem-Based Learning. \\ - Training. \\ - Medical Schools. \\ - Medical Faculty. \\ - Medical Education.}

Recebido em: 09/07/2011

Reencaminhado em: 26/11/2011

Reencaminhado em: 23/04/2012

Aprovado em: 11/05/2012

REVISTA BRASILEIRA DE EDUCAÇÃO MÉDICA
${ }^{I}$ Universidade CEUMA, São Luís, MA, Brasil

"Universidade Federal de São Paulo, São Paulo, SP, Brasil.

\section{RESUMO}

Foi feita uma revisão sistemática da literatura (1997-2007), no âmbito da formação docente, em currículos médicos orientados pela aprendizagem baseada em problemas, por meio de busca, identificação, seleção e análise de estudos nas bases Scielo, Lilacs, Cochrane, Medline, Ibesc, Pubmed e Eric com os descritores faculty OR faculty medical or staff development and education, medical or education, medical undergraduate or medical, schools and pbl. Os estudos analisados se agruparam em três eixos norteadores, que delinearam aplicações práticas para um programa de desenvolvimento docente eficiente: gestão participativa, maior investimento nos docentes, elaboração e aplicação de programas de educação continuada, permanente, com foco na construção dos conhecimentos, habilidades e atitudes.

\section{ABSTRACT}

Therefore we endeavor a systematic literature review (1997-2007) in the area of tutors graduation in doctors curriculum oriented in learning based on problems through: research, recognition, selection and study analysis on the data bases: Scielo, Lilacs, Cochrane, Medline, Ibesc, Pubmed and Eric within the description: faculty OR faculty medical or staff development and education, medical or education, medical undergraduate or medical, schools and pbl. From August to December 2009. The analyzed studies are defined in 3 axes that shaped practical applications for an effective tutor development program: participative management, greater investments in tutors, establishment and application of a constant learning program, permanent focused on knowledge building, skills and approaches. 


\section{INTRODUÇÃO}

Modificar a ação docente requer compreender o "sistema de crenças" dos professores e propor vivências que lhes permitam a sensibilização para transformarem, por meio de vivências, dinâmicas e experiências de vida que conscientizem a necessidade de se autossuperarem no saber-fazer pedagógico ${ }^{1}$.(p.41)

Tentativas de inserir mudanças significativas no processo da educação médica vêm sendo levadas a cabo no Brasil e no mundo. Ao longo dos anos, surgiu a necessidade de uma reflexão sobre as estratégias de desenvolvimento docente das escolas médicas que optaram por trabalhar com metodologia ativa em seu currículo. Assim, um processo de mudança foi incorporado, impondo-se quase como uma determinação, já que valoriza uma formação geral dos profissionais da área de Saúde 2 .

No Brasil, os questionamentos sobre o currículo convencional de Medicina somaram esforços a partir da década de 1990, com a Comissão Interinstitucional de Avaliação do Ensino Médico (Cinaem), que identificou deficiências na formação do médico. Em 1996, foi aprovada a Lei de Diretrizes e Bases da Educação Nacional (LDB), que extinguiu os departamentos e os currículos mínimos e propôs uma avaliação do ensino em todos os níveis. Já em 2001, as Diretrizes Curriculares Nacionais (DCN) de Medicina apresentaram competências necessárias a todas as áreas da Saúde e de cada curso específico, objetivando formar profissionais que pudessem compreender a integridade dos cuidados demandados pela população frente às novas tecnologias e ao desenvolvimento do Sistema Único de Saúde (SUS). As DCN de Medicina firmaram competências centradas no estudante, capaz de pensar criticamente, de analisar os problemas da sociedade e de procurar soluções para os mesmos, de forma a adquirir cada vez mais autonomia. Esse movimento de avaliação favoreceu uma reformulação curricular nas instituições ${ }^{3}$.

Pesquisadores têm apontado a necessidade de mudanças radicais nas bases das Políticas Públicas de Educação, afirmando que muitos são os docentes que redescobriram o prazer e a possibilidade de estabelecer vínculos no processo de ensino-aprendizagem. Esses docentes demonstram encantamento com o espaço da interdisciplinaridade, desafiados pela necessidade de mudar conceitos e práticas para dar conta das insuficiências de conhecimento que vão percebendo nessa trajetória ${ }^{3,4,5}$.

Com o projeto da Comissão de Avaliação das Escolas Médicas (Caem), afirma-se a relevância desta pesquisa. E se pensa: as novas escolas de ensino superior, que implantaram metodologias inovadoras, não teriam no desenvolvimento docente um desafio? Se não ocorrer o desenvolvimento docente como uma preocupação para implantação de novas propostas metodológicas, poder-se-á acreditar na implementação adequada desse currículo? Ao nos debruçarmos sobre a temática aqui apresentada, acreditamos que estas são algumas das questões sobre as quais os gestores e o corpo docente devem refletir.

A transferência do centro das ações de ensino para o aluno é um marco da pedagogia atual e um dos pressupostos da metodologia PBL $^{6}$. Nesse contexto, as novas organizações dos serviços de saúde do Brasil necessitam de profissionais com capacidade para articular prevenção, promoção e reabilitação. Médicos com formação geral, capazes de prestar uma atenção integral e individualizada às pessoas, que trabalhem em equipe, que saibam tomar suas decisões considerando não somente a situação clínica individual, mas também o contexto em que vivem os pacientes, recursos disponíveis e medidas mais eficazes $^{5}$

A decisão de muitas escolas de rever as metodologias de aprendizagem com base nas orientações das DCN e a necessidade de uma transformação significativa da formação dos profissionais de saúde, em especial da formação médica, nos levam à condução deste estudo, que tem por objetivo realizar uma revisão sistemática da literatura (RSL) no âmbito da formação docente em currículos médicos orientados pela aprendizagem baseada em problemas (ABP/PBL).

\section{METODOLOGIA}

A revisão sistemática da literatura (RSL) é um instrumento que pode identificar dados mensuráveis, confiáveis e consistentes para uma pesquisa científica, sendo a estratégia metodológica definida para esta pesquisa ${ }^{7}$. A pesquisa foi constituída de dois momentos: realização da RSL e análise temática dos achados selecionados no último critério de inclusão.

Foram pesquisados artigos publicados de 1997 a 2007 nas bases vinculadas à biblioteca virtual de Saúde (Bireme): Lilacs, Medline, Biblioteca Cochrane, Ibecs e Scielo. E na base de dados Eric (Education Resources Information Center) e Pubmed. A ferramenta MeSH foi utilizada como instrumento definidor dos descritores desta pesquisa. A partir dos ensaios preliminares na base de dados Pubmed, foram assumidos os descritores: faculty; faculty, medical; staff development; education, medical; education, medical, undergraduate; medical school; problem-based learning. Ressaltamos que o único limite de busca definido nesta pesquisa foram as bases de dados.

\section{Compondo o Campo Empírico}

Foram selecionados três momentos como critérios de inclusão e exclusão dos estudos: 
- foram definidos como ponto de corte os estudos que não apresentavam uma combinação de dois ou mais descritores previamente selecionados no planejamento da pesquisa. Os estudos que não cumpriam este critério foram excluídos da pesquisa. Em todas as bases de dados, até mesmo nas nacionais, optou-se por continuar com os descritores em sua forma original (MeSH-DeCS). Assim, minimizamos um viés na análise e seleção dos estudos. Iniciamos a aplicação dos critérios de exclusão com 442 estudos; após este momento, com a exclusão de 28 , restaram 414 ;

- a partir dos estudos incluídos, aplicou-se o segundo critério. Foi definido que, por meio dos resumos e/ou descritores dos achados, permaneceriam os artigos cujo tema incluísse: curso de Medicina, escola de Medicina, faculdade de Medicina, PBL/ABP. Todos os outros seriam descartados. Iniciamos este critério com 414, dos quais foram excluídos 322, restando 92;

- neste momento, foi definido que só permaneceriam na pesquisa os estudos que apresentassem "desenvolvimento docente nos cursos de Medicina fundamentados na metodologia de ensino ABP-PBL" como escopo da pesquisa. Assim, os estudos que não partilhavam desses critérios foram excluídos. O terceiro e último critério de inclusão se iniciou com 92 estudos, excluindo-se destes 75 .

Os estudos selecionados no terceiro critério de inclusão foram os estudos analisados. Nosso campo empírico, portanto, é composto por 17 estudos, cuja análise foi orientada pela questão da pesquisa: "como o desenvolvimento docente é trabalhado nas escolas médicas em currículos orientados pela ABP?"

Na exploração do material empírico, no que se refere aos conteúdos - características das estratégias e perspectivas e desafios para programas de desenvolvimento docente em currículos PBL, nos cursos de Medicina, localizados nos campos de metodologia, resultados, considerações ou conclusões do estudo - , esses conteúdos buscaram responder às seguintes perguntas:

- os autores caracterizam o desenvolvimento docente nas escolas médicas?

- os autores identificam no desenvolvimento docente se as práticas pedagógicas são reconhecidas pelos professores?

- os autores falam sobre possíveis contribuições para a elaboração de programas de desenvolvimento docente em currículos $\mathrm{ABP} / \mathrm{PBL}$ ?
Com base neste processo de identificação das unidades, o passo seguinte abrangeu a categorização dos dados, momento em que categorias e subcategorias emergiram nos conteúdos dos achados selecionados. A análise foi finalizada com a interpretação dos resultados.

\section{RESULTADOS}

Após mapeamento dos 442 estudos levantados na revisão, apenas 17 foram selecionados, analisados e caracterizados por ano, origem, periódico, base de dados e natureza do texto. $\mathrm{O}$ levantamento dos achados nas bases de dados Eric, Medline, Pubmed e Scielo deu-se no período de agosto a dezembro de 2009.

Os achados incluídos por ano demonstram um aumento na publicação de estudos voltados para o desenvolvimento docente nos cursos de Medicina baseados na metodologia PBL/ABP no ano de 2005, com leve queda em 2006. A partir de 2007, foi identificada uma perspectiva de incremento nos anos seguintes, com um aumento de mais de 50\% em 2008.

Quanto à origem, o maior número de publicações está nos Estados Unidos, representado por quatro estudos das publicações de 1980 a 2008, seguido por Canadá, Suíça e Brasil, com dois estudos cada. Os demais países aparecem apenas com uma publicação. Segundo Wetzel $^{8}$, vários programas de desenvolvimento docente surgiram com a origem do PBL/ABP. Estes programas foram elaborados e oferecidos pelos berços da metodologia. Contudo, é reconhecido que, apesar de já surgir um movimento para elaboração de programas de desenvolvimento docente em algumas escolas médicas, muitas delas não atentaram para a importância da existência e permanência desses programas.

Os achados apontam uma produção pouco significativa no que se refere ao desenvolvimento de professores nos currículos PBL. As bases de dados Lilacs e Cochrane não apresentaram estudos sobre a temática PBL. A Pubmed também apresentou um número de artigos reduzidos, com baixa produção em currículos PBL (uma publicação), mas traz várias publicações em desenvolvimento docente. Eric e Scielo apresentam igualmente um número reduzido de artigos sobre a temática (duas publicações cada). A base de dados Medline aparece com o maior número de publicações sobre desenvolvimento docente nos currículos PBL (12 publicações).

Os dois estudos nacionais, que abrangem relatos de experiência relacionada ao escopo desta revisão, sugerem também a necessidade de novos estudos para se aprofundar o que tem sido produzido em termos de pesquisa no Brasil sobre a temática investigada. A análise dos estudos permitiu identificar como núcleo central o reconhecimento dos programas de de- 
senvolvimento docente como um consenso entre os estudos analisados nesta revisão. Como pontua Evans", "em uma mudança curricular, o programa de desenvolvimento docente de professores tem um papel-chave a desempenhar" (p.366). Wet$\mathrm{zel}^{8}$ corrobora o fato, afirmando que um "eficiente programa de treinamento do corpo docente pode ser o ingrediente essencial do sucesso para inovação educacional" (p. 477). Já Almeida 10 "sustenta que a capacitação dos professores é imperiosa para currículos inovadores" (p. 241).

É importante destacar que não se utiliza uma só expressão para pesquisar/relatar/comentar sobre desenvolvimento docente. Em três estudos analisados, o termo comum empregado pelos autores é "capacitação"; em cinco dos estudos, é "treinamento"; e em sete, os autores usam a expressão "desenvolvimento docente". Em alguns estudos, os autores utilizam os termos "simpósio" e "workshop" como sinônimos dos termos acima referidos.

Um terceiro estudo abrange a significativa diversidade de estratégias de intervenção nos programas de desenvolvimento docente descritas nos estudos analisados, podendo ser identificadas e descritas 52 estratégias de ensino-aprendizagem apresentadas nesses programas. As que surgem com maior frequência são aplicação de questionário (sete eventos), fornecer feedback (seis eventos), sessões tutoriais (cinco eventos), discussão em grupo (três eventos), observação de vídeos e comportamentos, relato de experiência, dinâmica de grupo, princípios do PBL, enfrentamento das dificuldades, discussão teórico/conceitual (dois eventos cada).

Os diferentes achados analisados também foram delineados em três grandes eixos norteadores: características das estratégias utilizadas nos programas de desenvolvimento; desafios dos programas; perspectivas destes programas.

No campo analítico-interpretativo, seis categorias representam o primeiro eixo:

\section{Mediações e Trocas se Subdividem em Heteroavaliação Participação de Diferentes Atores e Interdisciplinaridade}

A participação de diferentes atores na investigação surge como o segundo ponto mais discutido nos achados. Quatro dos estudos apresentam estudantes, consultores e pedagogos como chave para um programa de desenvolvimento docente efetivo. Os estudantes surgem ora como indicadores da qualidade do ensino dos tutores, ora como participantes dos treinamentos, apontando dificuldades e facilidades dos membros docentes. Os consultores e pedagogos surgem ou como convidados ou como participantes de um programa já existente ou na elaboração e acompanhamento de um novo programa, emergindo nos achados como modelos que, por terem viven- ciado situações semelhantes às assinaladas pelos professores / participantes como situações difíceis, apresentam formas mais adequadas de trabalho nessas situações de conflito. Entendemos que os diferentes atores que contribuem nesse processo se situam como fator substancial para a eficácia do programa.

Colocar o tutor mais experiente como referência do tutor iniciante é o que sugere o núcleo temático 5 (tutores experientes como aporte do iniciante). Nos achados, este item coloca o tutor ora como líder do grupo tutorial, que será observado pelos tutores iniciantes, ora tutoriando os próprios ou, até mesmo, como seu suporte nas reuniões tutoriais ${ }^{11}$. "O melhor desenvolvimento docente se dá de tutor para tutor, os mais experientes oferecem soluções para os problemas comuns das tutorias $^{\prime \prime}$ (p.32). Essa temática se refere estritamente ao processo da avaliação ao final do programa no olhar dos avaliadores. Em sua totalidade, o item é avaliado como positivo sempre que nasce na análise dos artigos "percebemos que os participantes do simpósio melhoraram seus conhecimentos e atitudes frente à metodologia de forma significativa"12 (p.75).

\section{Temas Significativos para a Realidade do Professor}

Essa categoria demonstra que nove dos achados selecionados têm objetivos predefinidos, e cinco destes reforçam a ideia de trabalhar com programas fechados, nos quais os formadores apresentam os objetivos do curso como o primeiro passo para iniciar o programa.

Quatro manuscritos analisados situam a construção de programas com base em pesquisas desenvolvidas como o primeiro passo para levantar as necessidades reais dos professores. Assim, apontam uma nova forma de construir o treinamento ou programa de desenvolvimento de professores. Os autores expressam a importância de buscar as reais necessidades ou dificuldades do corpo docente e, após identificá-las, construir o programa com base nelas. Cerca de $70 \%$ dos professores foram avaliados pelos estudantes como insuficientes em fornecer feedback e apenas 3\% dos professores identificaram essa técnica como algo que deva ser treinado ${ }^{13}$. Os autores denunciam que as áreas em que os professores precisam de melhoria não estão sendo identificadas.

\section{Avaliação: o Lugar do Feedback}

A avaliação também se subdivide em avaliação no final do treinamento docente, no início e final do treinamento e avaliação do processo (diária). Também surgem, diferentes ferramentas de avaliação alguns pesquisadores referem trabalhar com aplicação de questionários, outros como inquérito e ainda outros, uma escala de Likert. Cerca de 53\% dos trabalhos avaliaram seus resultados diariamente ou ao final do processo 
com ferramentas como inquéritos ou questionários. Um dos autores afirma que "o professor pode ser avaliado antes ou depois da participação no programa e essa ferramenta deve seguir regras claras, metas, validação, confiança e eficiência, sendo um processo importante" 14 .

Essa categoria apresenta sete dos estudos analisados e que se subdividem pela singularidade de cada achado. $\mathrm{O}$ feedback surge ora como técnica utilizada para levar o professor a refletir sobre sua prática, ora como ferramenta a ser treinada e utilizada pelos professores em suas funções. Segundo Baroffio et al. ${ }^{13}$, "o feedback deve ser considerado um componente essencial de aprendizagem, pois reforça conhecimentos e habilidades adequadas e corrige possíveis erros" (p.366). Em um dos documentos analisados, os argumentos apresentados sustentam que, para habilitar o professor/tutor a se desenvolver, será necessário que o feedback aconteça e tenha caráter informativo, formativo, válido e confiável. Não é suficiente fornecer feedback, sua qualidade é o mais relevante ${ }^{15}$.

\section{Cenários de Vivência e Sensibilização Subdividindo-se em Integrar Diferentes Dimensões}

Cenários de vivência e sensibilização se identificam com os diferentes ambientes de ensino-aprendizagem que os professores experienciavam durante os treinamentos. Abrangeram sessões de debrief com estudantes e professores, coordenados pelos consultores e sessões tutoriais em que tutores tutoriavam ou eram tutoriados. Dramatizações e simulações também eram utilizadas nesses momentos com os tutores para esclarecer sobre seu papel e sobre como fornecer feedback. Técnicas interativas de ensino também eram usadas nos treinamentos, como roleplays e dinâmica de grupo. Não só o conteúdo do curso de desenvolvimento profissional, mas também os métodos de ensino usados contribuíram para o sucesso do curso. Foram modeladas as habilidades que se queria ensinar por meio de um treinamento interativo ${ }^{16}$. Nos diferentes achados, a simulação dos cenários tutoriais surge como espaço útil de aprendizado, espaços estes que propiciam os docentes, oportunidade de vivenciar um tutorial, discutir em grupo, brainstorms, estes, são pontos positivos dos treinamentos. O Programa de desenvolvimento externo e contratação de um olhar externo também surgem nos achados, como uma possibilidade para os programas de desenvolvimento docente.

\section{Obrigatoriedade da Participação em Programas de Formação Docente}

Os estudos selecionados apresentam o assunto como obrigatório ora para professores iniciantes, ora para todos os professores. Quando o programa se apresenta em dois módulos, o pri- meiro é obrigatório, e o segundo é opcional. Não existe uma singularidade nos programas analisados. Entretanto, um dos manuscritos afirma que "o fato de não ser obrigatório torna o programa um cenário motivador e sugestivo para o aprendizado do professor que o busca"17 (p.76).

\section{Autoavaliação e Reflexão}

A segurança na prática profissional se reflete na comparação entre as avaliações aplicadas antes e após o treinamento. Os sujeitos da pesquisa, os professores, afirmam maior segurança e confiança em suas atitudes após a participação nos programas de desenvolvimento docente. Todavia, um dos estudos aponta uma incongruência entre a autoavaliação do professor e seus comportamentos observados. Ao investigarem as autoavaliações e observarem suas dinâmicas, os autores identificam "diferenças significativas, que os levam a perceber a falta de reflexão nas atividades. Como hipótese para este dado, há um curto tempo entre as informações do treinamento e sua prática" $^{\prime 18}$ (p.1051).

Em média, os programas de desenvolvimento docente têm duração de dois a cinco dias e, em sua maioria, tempo integral. Os tipos de estudo que prevalecem nos achados selecionados são pesquisas que, após análise dos dados, orientam mudanças comportamentais do professor, estudos que originalmente tratam de desenvolvimento docente e pesquisas que servem como norte para a elaboração destes.

No campo analítico-interpretativo a seguir, sete categorias representam o segundo eixo.

\section{Articulação entre Teoria e Prática}

Muitas vezes, a divergência entre a fala e a prática se revela por uma incongruência entre a autopercepção do professor e sua fala ou comportamento. Ao final do processo, na avaliação do programa, os professores relatam que este foi importante para o seu desenvolvimento profissional e para a integração entre eles. A escolha de ferramentas de ensino interativas possibilitou a melhoria das habilidades como facilitador e condutor de pequenos grupos. Contudo, após o programa, os professores relatam não entender a função e o papel do tutor ${ }^{19}$. Entretanto, esta percepção dos professores não se confirma nas avaliações dos estudantes. Os autores comentam, ao final do estudo, que, um ano depois, o grupo de professores que passou pelo treinamento obteve uma melhora nas avaliações dos estudantes ${ }^{13}$.

\section{Implementar Mudanças}

Ênfase no programa de desenvolvimento docente ocorre em 16 dos estudos analisados. Aborda quatro principais temas: o 
programa, reflexão na ação, melhora no desempenho e tempo. O programa de desenvolvimento surge em sete dos estudos analisados e se caracteriza como essencial ora na transição curricular, ora na implantação, ora na impressão do papel do tutor mais experiente e dos iniciantes, e, até mesmo, no desenvolvimento de novas habilidades de ensino.

Para Pandya ${ }^{16}$, "a não ser que os professores sejam treinados e conscientizados [...] percebemos que a inovação na educação em Saúde não pode ser implementada" (p.246). O tempo dos docentes é um dos fatores que influenciam essa dinâmica de treinamento ${ }^{15}$.

Os autores recomendam que os programas de desenvolvimento docente sejam organizados no planejamento das escolas médicas, nas mudanças curriculares ${ }^{19}$. A Cinaem identificou problemas/dificuldades na formação do médico egresso, no corpo docente e no modelo pedagógico adotado pelos cursos. Neste sentido, surgiram iniciativas destinadas a impulsionar, apoiar e consolidar as mudanças no ensino médico, tais como o Programa de Incentivo às Mudanças Curriculares das Escolas Médicas (Promed) e o Programa Nacional de Reorientação da Formação Profissional em Saúde (Pró-Saúde). Apesar do incentivo, as escolas que iniciaram suas atividades com a implantação de novas metodologias de ensino têm um desafio importante a vencer: a formação docente para as diversas funções demandadas pelos novos currículos ${ }^{3}$.

\section{Compatibilizar as Demandas com o Tempo de cada Professor}

Poucos estudos trazem reflexões sobre esta categoria. Ela emerge como um processo que acontece após o programa, estando vinculado a uma avaliação que indicaria pontos positivos e negativos da prática em relação ao que foi vivenciado no treinamento. Os formadores observam, a longo prazo, também por meio das avaliações, os mesmos indicadores. "Os estudos que apresentam essa estratégia reforçam que o tempo entre os professores assimilarem as informações do treinamento e suas vivências deve ser respeitado"19 (p.1052). Neste sentido, Baroffio pontua que "após o programa, os professores precisam ter um tempo para pôr em prática, autorrefletir e incorporar as novas estratégias de ensino para melhorar o desempenho"13 (p.438).

\section{Sustentabilidade (Condições Pessoais/Institucionais)}

Segundo os dados analisados nos achados, o termo "sustentabilidade" vem reforçar e agrupar os termos "efetividade" e "eficácia" para a elaboração e o desenvolvimento dos programas de desenvolvimento docente. Para isso, há duas condições: apoio da instituição e motivação pessoal do professor ${ }^{15}$.

\section{Valorização da Docência}

Ramani ${ }^{14}$ apresenta temas abordados em uma publicação do Conselho Britânico de Medicina que trata dos médicos do futuro. Estes devem reconhecer a responsabilidade de ensinar, reconhecer que o ensino não é inato, mas poderá ser aprendido e que o exemplo do professor é uma influência importante na formação do estudante. Neste mesmo estudo, o autor reforça a importância dos programas de desenvolvimento docente e afirma que estes deverão ser elaborados com base nas necessidades da instituição e dos professores ${ }^{11}$.

\section{Papel do Docente}

Os estudos abordam a falta de formação formal dos professores e suas inadequações ao ensino quando eles têm de assumir diferentes funções - gestor, facilitador de pequenos grupos, consultor, pesquisador sem suporte didático-pedagógico. Muitas dessas funções se tornam desmotivantes para a grande maioria dos docentes ${ }^{14}$.

Ênfase no papel do tutor é uma das categorias que se apresenta em $50 \%$ dos estudos analisados neste segundo eixo e que se subdivide pela singularidade de cada achado. São 17 subtemas que se traduzem em pontos delineados para adequação e melhoria da prática docente, que perpassam pelos temas currículo, ensino-aprendizagem, avaliação e formação. Para que o tutor imprima seu papel, é importante a continuidade dos treinamentos, tanto dos novos tutores quanto dos experientes ${ }^{15}$.

\section{Desvalorização do Ensino}

Esta categoria surgiu em grande parte dos estudos analisados, trazendo a falta de formação formal ou didático-pedagógica dos médicos professores como a principal causa da desmotivação e desvalorização do ensinar. O processo de implantação, implementação ou mudança curricular requer uma abordagem profissional e acadêmica de ensino-aprendizagem, mas os professores necessitam de suporte e treinamento para que se dediquem ao ensino como o fazem em relação à pesquisa ${ }^{15}$. A promoção de uma cultura institucional que valide o ensino de excelência levará o ensino e a pesquisa a participarem de um mesmo nível de relevância ${ }^{14}$.

O terceiro e último eixo é representado por três categorias em seu campo analítico-interpretativo, representadas a seguir.

\section{Espaço de Reflexão na Prática Docente}

As mudanças curriculares nos cursos de Medicina no País e no mundo são recentes, mas o desenvolvimento das práticas docentes é necessário para a adequação desses novos currículos. Os dados indicam que a reflexão sobre as práticas docentes como um processo contínuo e de desconstrução surgiu como uma alternativa no campo da formação do professor em Saúde. 
Para Baroffio ${ }^{13}$, após os programas de desenvolvimento, os professores precisam ter um tempo para pôr em prática e autorrefletir, para que possam incorporar as novas estratégias de ensino e melhorar seu desempenho. Isto vem se confirmando com o bom resultado da avaliação após um ano do treinamento.

\section{Continuidade}

Segundo sugere a literatura, os docentes devem ter maior qualificação, desenvolvimento constante e contínuo, e reflexão e prática em novas estratégias de ensino muito além do conteúdo disciplinar ensinado. A aprendizagem baseada em problemas ensejará possibilidades de contato pessoal entre os docentes, permitindo maior integração curricular. A experiência do programa de desenvolvimento docente possibilitou trocar experiências e minimizou a angústia e as debilidades de quem está se iniciando como professor/tutor. A formação permanente dos professores será primordial em programas que adotarem a inovação pedagógica como referência educacional ${ }^{11}$.

\section{Política de Qualidade para a Educação Médica}

A maioria das escolas de Medicina reconhece a necessidade da ajuda de educadores profissionais que estejam familiarizados com o campo da Medicina. Os programas de desenvolvimento surgem, então, como um ingrediente essencial para o sucesso da inovação educacional. Cada escola, segundo Wetzel ${ }^{8}$, deverá trabalhar os detalhes para satisfazer suas próprias metas, objetivos e cultura.

\section{CONCLUSÃO}

Esta pesquisa teve os objetivos de caracterizar o desenvolvimento docente na escola médica que assume um currículo orientado pela metodologia ABP/PBL; identificar no desenvolvimento docente as práticas pedagógicas reconhecidas pelos docentes; e mapear possíveis contribuições para a elaboração de programas de desenvolvimento docente em escolas médicas com currículo ABP/PBL.

Na análise dos achados, identificou-se que não há um padrão para caracterizá-los, mas que existe uma singularidade neste fazer. As práticas pedagógicas seguem o mesmo desdobramento das características e remontam, de acordo com suas expectativas, às estratégias de ensino, que, muitas vezes, são assumidas pelos participantes ao final do processo. Identificou-se que a importância do desenvolvimento docente foi reconhecida em todos os artigos selecionados.

A temática é de suma importância para o desenvolvimento e aprimoramento da educação médica, constituindo um grande desafio para a produção científica nacional e internacional. Foram apontadas aplicações práticas encontradas nesta pesquisa para um programa de desenvolvimento docente eficaz e eficiente: gestão participativa, maior investimento nos docentes, elaboração e aplicação de programas de educação continuada, permanente, com foco na construção dos conhecimentos, habilidades e atitudes.

Mais do que identificar dificuldades ou limitações, é preciso abrir caminhos que possam mostrar que há um processo de mão dupla no aprender e ensinar em Medicina, articulando-o com um projeto maior de formação em saúde, comprometido com a consolidação do SUS e com os profissionais envolvidos nas demandas sociais.

\section{REFERÊNCIAS}

1. Campos CM. Saberes docentes e autonomia dos professores. 2 ${ }^{\mathrm{a}}$ ed. Petrópolis: Vozes; 2009.

2. Mamede S, Penaforte J, orgs. Aprendizagem baseada em problemas: anatomia de uma nova abordagem educacional. Fortaleza: Hucitec; 2001. p.231.

3. Batista SHS. A Interdisciplinaridade no Ensino Médico. Rev bras educ méd. 2006;30(1):39-45.

4. Perim GL, Abdalla IG, Aguilar-da-Silva RH, Lampert JB, Stella RCR, Costa NMCS. Desenvolvimento docente e a formação de médicos. Rev bras educ méd. 2009; 33 (supl.1). [capturado 14 dez. 2009). Disponível em: <http://www. scielo.br / scielo.php?script=sci_arttext\&pid=S0100$-55022009000500008 \& \operatorname{lng}=$ en\&nrm $=$ iso $>$.

5. Feuerwerker L. Além do discurso de mudança na educação médica: processos e resultados. São Paulo: Hucitec; Londrina: Rede Única; Rio de Janeiro: Associação Brasileira de Educação Médica; 2002.

6. Demo P. Aula não é necessariamente aprendizagem. Ensaio: Avaliação e Políticas Públicas em Educação. Rio Comprido. 2004;12(43):669-695.

7. Atallah ÁN. Revisão sistemática da literatura médica e metanálise. Diagnóstico \& Tratamento. 1997;2(2):12-15.

8. Wetzel MS. Developing the role of the tutor/facilitator. Postgraduate Medical Journal. 1996;72(850):474-477.

9. Evans PA, Taylor DC. Staff development of tutor skills for problem-based learning. Medical Education. 1996;30(5): 365-6.

10. Almeida HGG, Ferreira Filho OF. Educação permanente de docentes: análise crítica de experiências não sistematizadas. Rev bras educ méd. 2010;32(2) [capturado 30 ago. 2010] Disponível em: <http://www.scielo.br/scielo. php?script=sci_arttext\&pid=S0100-55022008000200012\& lng=en\&nrm=iso $>$.

11. Faria MJSS, Nunes EFPA, Anastaciou L, Sakai MH, Silva VLM. Os desafios da educação permanente: a experiência do curso de Medicina da Universidade Estadual de Londrina. Rev bras educ méd. 2008; 32(2):248-253. 
12. Rahman ME. Knowledge and attitude of faculty members on problem based learning. Mymensingh Medical Journal. 2004; 13(1): 20-4.

13. Baroffio A, Nendaz MR, Perrier A, Vu NV. Tutor Training, Evaluation Criteria and Teaching Environment Influence Students' Ratings of Tutor Feedback in Problem-Based Learning. Advances in Health Sciences Education. 2007; 12(4):427-439.

14. Ramani S. Twelve tips to promote excellence in medical teaching. Medical Teacher. 2006; 28(1):19-23.

15. Van Wyk J, Mclean, M. Maximizing the value of feedback for individual facilitator and faculty development in a problem-based learning curriculum. Medical Teacher. 2007; 29(1):26-31.

16. Pandya H, Ghosh S. Sensitizing faculty to the problem-oriented approach as an instructional method: experience of a brief faculty development workshop. National Medical Journal of India. 2008; 21(5):243-5.

17. Baroffio A, Kayser B, Vermeulen B, Jacquet J, Vu NV. Improvement of tutorial skills: an effect of workshops or experience? Academic Medicine. 1999; 74(suppl. 10): S75-7.
18. Tipping J, Freeman RF, Rachlis AR. Using faculty and student perceptions of group dynamics to develop recommendations for PBL training. Academic medicine. 1995; 70(11):1050-2

\section{CONTRIBUIÇÃO DOS AUTORES}

As autoras orientaram os caminhos percorridos durante a pesquisa, desde sua estrutura metodológica até a análise e conclusão dos dados consolidados.

\section{CONFLITO DE INTERESSES}

Declarou não haver.

\section{ENDEREÇO PARA CORRESPONDÊNCIA}

Cinthia Rêgo

Av. dos Holandeses, 1103 Cond. Toscana Edf. Clássico Apto 302

Ponta do Farol - São Luís

CEP 65075-650 - MA

E-mail: cinthia_fernanda@hotmail.com 